\title{
Intelligent OS Process Scheduling Using Fuzzy Inference with User Models
}

\author{
Sungsoo Lim and Sung-Bae Cho \\ Dept. of Computer Science, Yonsei University \\ Shinchon-dong, Seodaemun-ku, \\ Seoul 120-749, Korea \\ lss@sclab.yonsei.ac.kr, sbcho@cs.yonsei.ac.kr
}

\begin{abstract}
The process scheduling aims to arrange CPU time to multiple processes for providing users with more efficient throughput. Except the class of process set by user, conventional operating systems have applied the equivalent scheduling policy to every process. Moreover, if the scheduling policy is once determined, it is unable to change without resetting the operating system which takes much time. In this paper, we propose an intelligent CPU process scheduling algorithm using fuzzy inference with user models. It classifies processes into three classes, batch, interactive and real-time processes, and models user's preferences to each process class. Finally, it assigns the priority of each process according to the class of the process and user's preference through the fuzzy inference. The experimental result shows the proposed method can adapt to user and allow different scheduling policies to multiple users.
\end{abstract}

Keywords: OS process scheduling, fuzzy inference system, process classification, user's preference.

\section{Introduction}

Recent years there have been significant efforts in applying the reflection paradigm in system software. Reflection is widely acknowledged as a useful mechanism for facilitating the run-time adaptation and reconfiguration of software. This approach has been particularly directed at middleware systems [1], but there has also been significant work in reflective operating systems [2]. In this paper, we propose an intelligent process scheduling algorithm in operating systems.

The goal of process scheduling is to give users more efficient throughput by arranging CPU time to multiple processes. However, it is hard to arrange CPU time fairly because the purpose of each process is different. In general, there are three different purposes according to the classes of processes. The first class is batch processes such as programming language compilers, database search engines and scientific computations. They require high throughput and short turn-around time. The second one is interactive processes such as command shells, text editors and graphical applications. They interact constantly with their users and thereby spend a 
lot of time waiting for key-presses and mouse operations. This makes them to require short response time to user input. The last one is real-time processes such as video and sound applications, and robot controllers. They should have a short guaranteed response time with a minimum variance [3].

Since there are different purposes according to the classes of processes and the conventional operating systems do not know the classes of processes, the research at process scheduling has proposed for a specific purpose of scheduling or for working efficiently in specific environment. In this paper, we propose a novel method of process scheduling which adapts to users' preferences. It extracts the features of processes in run time, and classifies the classes of processes. It models the preferences of users, and finally decides the priority of each process using fuzzy inference with the information of process class and user's preferences.

\section{Related Works}

\subsection{Process Scheduling}

In general, the operating systems which allow multiple processes focus on the problem of how to arrange CPU resource to processes. One of the oldest, simplest and most widely used proportional share scheduling algorithms is round robin [4]. It is the time-sharing approach in which several tasks can coexist. The scheduler gives a short time-slice to each job, before moving on to the next job, polling each task round and round. This way, all the tasks advance, little by little, on a controlled basis. There are other traditional methods, which are based on CPU resource, such as FCFS (first come first served), SJF (shortest job first), SRT (shortest remaining time), HRRN (highest response ratio next) and so on [5].

Recently, through the enhancement of CPU computational power and network bandwidth, there are some studies on CPU scheduling to support multimedia tasks. Yavatkar and Lakshman use a rate adjustable priority scheduling mechanism to provide an average frame delivery rate to a client application [6]. SMART proposed by Nieh and Monica is a hierarchical CPU scheduling algorithm that supports both hard real time and conventional time sharing applications, adjusts well to overload, and can notify applications when their deadlines cannot be met [7]. Other scheduling work has been done in supporting clients with real-time requirements [8,9] and improving the response time of interactive clients $[10,11]$.

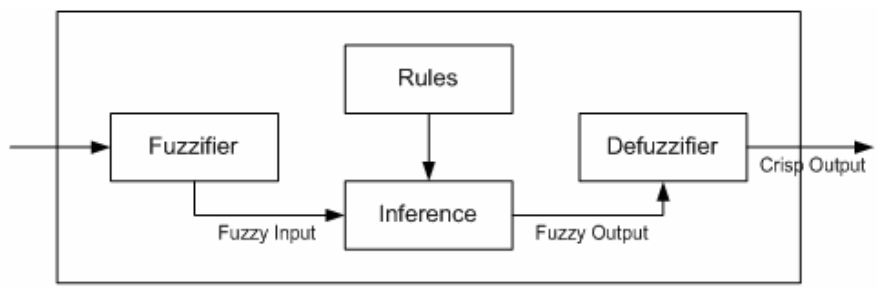

Fig. 1. Fuzzy inference system 


\subsection{Fuzzy Inference System}

Fuzzy inference systems (FIS), which are also called fuzzy rule-based systems, are composed of 4 blocks as shown in Fig. 1. Fuzzifier transforms the crisp inputs into fuzzy inputs by membership functions that represent fuzzy sets of input vectors. In this paper, we adopt the triangular membership functions. Rule-base consists of fuzzy IF-THEN rules. Inference is an inference engine for fuzzy rules. In this paper, we use max min composition rules for inference method. Defuzzifier transforms the fuzzy output into crisp output. Defuzzification process causes the most computational complexity in FIS. We apply weighted average for defuzzification.

$$
R_{l} \text { : if } x_{1}=F_{1}^{l} \text { and } x_{2}=F_{2}^{l} \text { then } y=Q_{k}^{l}
$$

For instance, let the $l$ th rule as (1), where $x_{i}$ denotes the input variable and $F$ and $Q$ denote the fuzzy set of input and output variables. Fig. 2 shows the inference process with the $l$ th fuzzy rule using triangular membership functions and $\max \cdot \min$ composition.

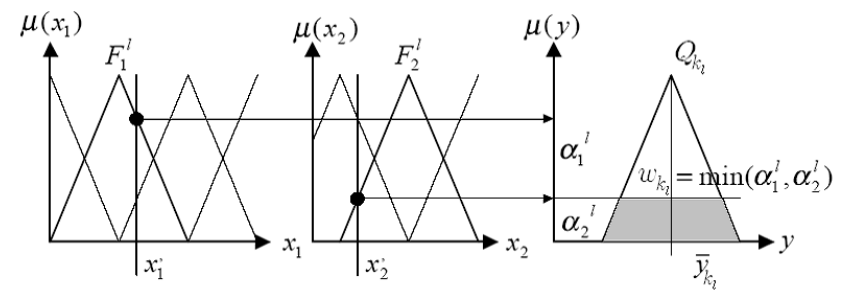

Fig. 2. The process of fuzzy inference

By the fuzzy inference with the lth fuzzy rule, we obtain the result through $\min$ operation as the shady part in Fig. 2. After applying all the rules, we can get the final result of fuzzy inference through max operation as follows:

$$
Q_{k}=\max \left(\omega_{k_{1}}, \omega_{k_{2}}, \cdots, \omega_{k_{n}}\right) \quad(n \text { is the total number of rules) }
$$

This is followed by the last step of FIS, defuzzification, with weighted average.

$$
y(x)=\frac{\sum_{k=1}^{n} \bar{y}_{k} \times Q_{k}}{\sum_{k=1}^{n} Q_{k}}
$$

Here, $\bar{y}_{k}$ represents the value of $y$-axis when the membership value of fuzzy set $Q_{k}$ is 1.0 .

In this paper, we apply fuzzy inference to process classification and process priority decision. FIS has been applied to numerous engineering applications such as control, signal processing and pattern classification problems [12,13]. In pattern classification, fuzzy logic improves classification and decision systems by allowing the use of overlapping class definitions and improves the interpretability of the results by providing more insight into the classifier structure and decision-making systems [14]. Moreover, the function schedule() is called by OS in every brief time duration, 
and we apply fuzzy inference because it requires low computational overhead comparing with other artificial intelligence methods.

\section{Intelligent Process Scheduling}

As shown above, although the purpose of process scheduling is different according to the classes of process, the conventional operating systems have scheduled every process equivalently because they do not know the classes of process. To overcome this limitation, we develop an intelligent process scheduling method in Linux kernel 2.4.25. The method consists of three modules as shown in Fig. 3. The process classification module classifies a process into batch, interactive and real-time processes with the type and frequency of system call and the amount of CPU time which the processes are used. The user modeling module models the user's preference about the three classes of processes. The process priority module decides the priority of each process using the result of classification and user models.

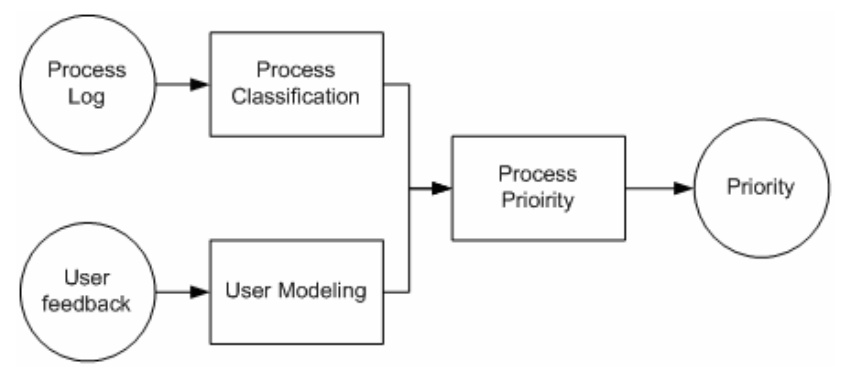

Fig. 3. Proposed process scheduling method

\subsection{Process Classification}

Fig. 4 shows the entire process of the process classification. For the process classification, we produce a log by pairing the occupation time of CPU and the type of system call together, where the occupation time of CPU denotes the CPU time which the process uses between the last log and the current one.

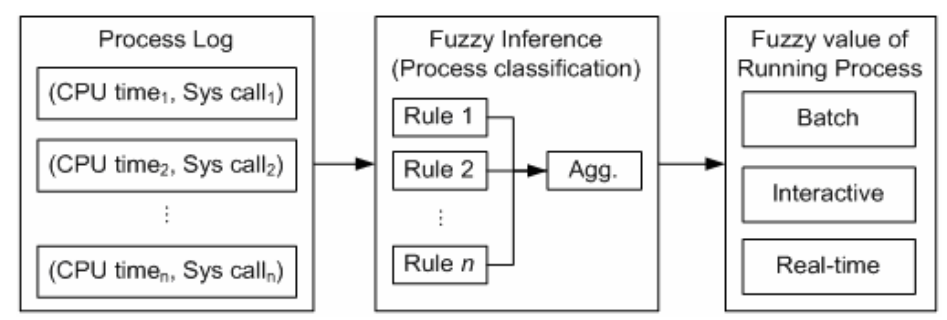

Fig. 4. Fuzzy process classifier 
A queue is inserted in task_struct to record the recent $n \operatorname{logs}(n=100)$. When the process is scheduled out or system call is occurred, a pair of logs is pushed in the quеие. If the quеие is full, it pops a log before pushing. When the process is scheduled out, the null value is inserted for the system call value.

To calculate CPU time, we add a variable, bf_counter, in task_struct for remembering the counter value before the process gets CPU control, where the counter variable in task_struct denotes the remaining arranged CPU time for the process. When the process is scheduled out or blocked by system call, we can get the used CPU time of the process from the difference of these two variables.

As shown in Fig. 4, we use the process log data for input value of fuzzy inference and we get the membership values about process classes through the fuzzy inference. The fuzzy rules are made by analyzing each class of processes. The batch processes, like programming language compilers and database search engines, require more CPU time than other classes of processes, and the interactive processes such as documents editors interact with humans through input and output devices they have more system calls related on input and output than others. The real-time processes have more sys_nanosleep () system calls than others for giving CPU control to other processes after finishing their computations. The fuzzy inference for classifying a process is conducted when a new log comes for the process in order to maintain the present condition of the process and to prevent duplicate calculations. Table 1 shows the fuzzy rules for classifying processes and Fig. 5 represents the membership functions for it.

Table 1. Fuzzy rules for classifying processes

\begin{tabular}{ll}
\hline \multicolumn{1}{c}{ Condition } & \multicolumn{1}{c}{ Action } \\
\hline if system call $\{$ sys_read ()$\}$ is many, & then interactive process \\
\hline if system call $\{$ sys_write ()$\}$ is many, & then interactive process \\
\hline if CPU using times (per ticks) are many, & then batch process \\
\hline if system call $\{$ sys_nanosleep ()$\}$ is many, & then real-time process \\
\hline
\end{tabular}

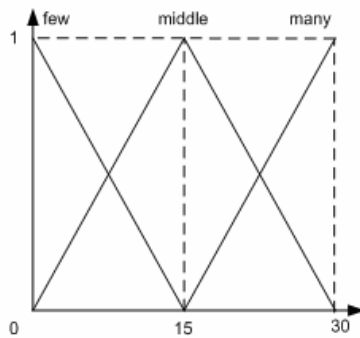

(a) sys_nonosleep, CPU using time

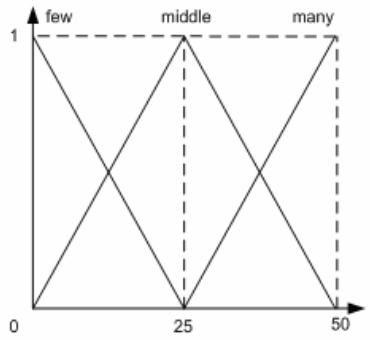

(b) sys_read(), sys_write()

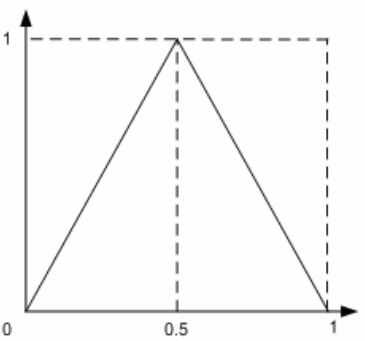

(c) interactive, batch and real-time process

Fig. 5. Membership functions for classifying processes 


\subsection{Decision of Process Priority}

Conventional operating systems have scheduled processes on the system level without considering user's preferences. Therefore, if user wants to change the process scheduling policy, he/she must be reset the OS which needs much time and is hard for novice.

In this section, we introduce a user adaptive process scheduling method by changing the priority of process according to the user's preferences. With this method, an operating system can satisfy multi-users' needs without resetting the operating system. It only requires modeling the user's preferences. Fig. 6 shows the process of decision of process priority based on user model and the result of process classification.

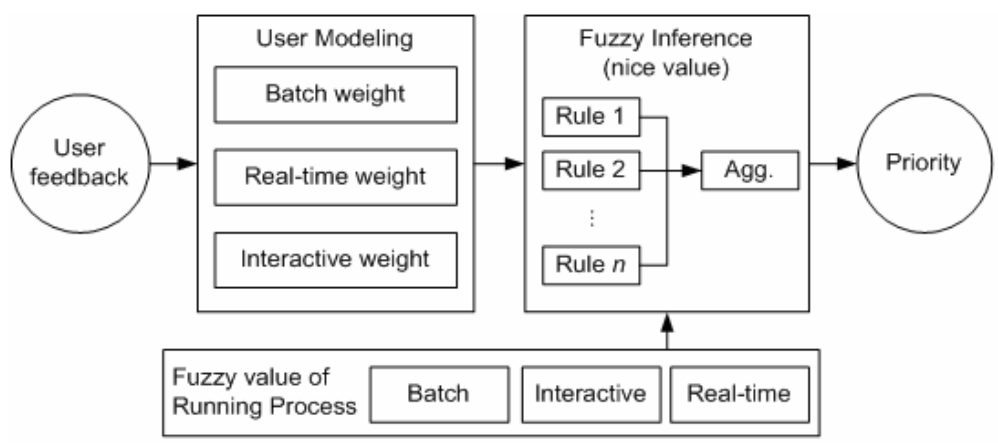

Fig. 6. Decision of process priority

In Linux, the task_struct has a pointer of user_struct to denote who the owner of this process is. Therefore, we insert variables for user's preferences in the user_struct. When the user is logged out after using the computer, we get the user feedback about the amount of satisfaction to each class of process. The user inputs the score from 0 to 10, and then the system calculates the preferences of the user like Table 2. We design the update method focused on four factors. First, if the feedback value of a class is low, it means that user wants to arrange more CPU time for the class of processes, and on the other hand if the feedback value of a class is high, it denotes that user wants to arrange less CPU time for the class of processes. Second, the summation of three weights $\left(\omega_{\text {batch }}+\omega_{\text {inter }}+\omega_{r t}\right)$ must be 1 . Third, if user inputs the same three values, the weights do not change. Last, the amount of updates depends on the relative gaps of the user's input, not the absolute gaps.

Except setting up as real-time processes by user, the processes in Linux are applied SCHED_OTHER schedule policy, which plays a role in round robin, and the priority of processes is deeply related on the value of nice in task structure. If the value of nice is high, it denotes the process has low priority, on the other hand, if its value is low, it means the process has high priority. Therefore, we determine the value of nice to control the priority of processes. 
Table 2. Update of user's preferences from feedback

\begin{tabular}{lll}
\hline$M=\frac{f_{\text {batch }}+f_{\text {inter }}+f_{\text {rt }}}{3}$ & $S=T_{\text {batch }}+T_{\text {inter }}+T_{\text {rt }}$ & \\
$T_{\text {batch }}=\omega_{\text {batch }} \times\left(1-\frac{f_{\text {batch }}-M}{M}\right)$ & $\omega_{\text {batch }}=\frac{T_{\text {batch }}}{S}$ & $\omega:$ weight, \\
$T_{\text {inter }}=\omega_{\text {inter }} \times\left(1-\frac{f_{\text {inter }}-M}{M}\right)$ & $\omega_{\text {inter }}=\frac{T_{\text {inter }}}{S}$ & $f:$ feedback \\
$T_{\mathrm{rt}}=\omega_{\mathrm{rt}} \times\left(1-\frac{f_{\mathrm{rt}}-M}{M}\right)$ & $\omega_{\mathrm{rt}}=\frac{T_{\mathrm{rt}}}{S}$ & \\
\hline
\end{tabular}

Table 3. Fuzzy rules for the decision of process priority

\begin{tabular}{|c|c|}
\hline Condition & Action \\
\hline $\begin{array}{l}\text { if (process is interactive process) and (user } \\
\text { interactive weight is high), }\end{array}$ & then nice is low \\
\hline $\begin{array}{l}\text { if (process is interactive process) and (user } \\
\text { interactive weight is middle), }\end{array}$ & $\begin{array}{l}\text { then nice } \\
\text { middle }\end{array}$ \\
\hline $\begin{array}{l}\text { if (process is interactive process) and (user } \\
\text { interactive weight is low), }\end{array}$ & then nice is high \\
\hline $\begin{array}{l}\text { if (process is batch process) and (user batch } \\
\text { weight is high), }\end{array}$ & then nice is low \\
\hline $\begin{array}{l}\text { if (process is batch process) and (user batch } \\
\text { weight is middle), }\end{array}$ & $\begin{array}{ll}\text { then } & \text { nice } \\
\text { middle } & \\
\end{array}$ \\
\hline $\begin{array}{l}\text { if (process is batch process) and (user batch } \\
\text { weight is low), }\end{array}$ & then nice is high \\
\hline $\begin{array}{l}\text { if (process is real-time process) and (user real- } \\
\text { time weight is high), }\end{array}$ & then nice is low \\
\hline $\begin{array}{l}\text { if (process is real-time process) and (user real- } \\
\text { time weight is middle), }\end{array}$ & $\begin{array}{l}\text { then nice } \\
\text { middle }\end{array}$ \\
\hline $\begin{array}{l}\text { if (process is real-time process) and (user real- } \\
\text { time weight is low), }\end{array}$ & hice is \\
\hline
\end{tabular}

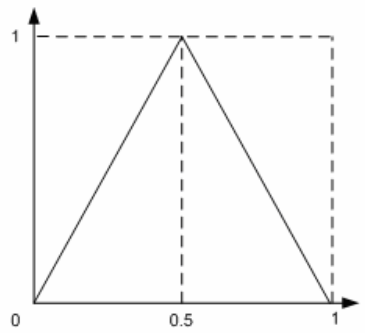

(a) interactive, batch and real-time process

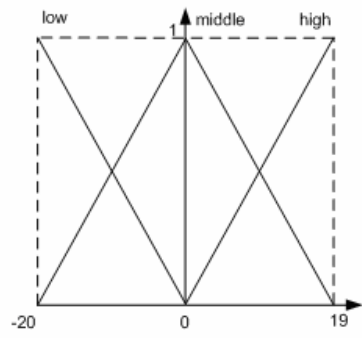

(b) nice

Fig. 7. Membership functions for the decision of process priority 
With the user's preferences and the fuzzy membership values of process which explained at 3.1, we can determine the value of nice through fuzzy inference. Table 3 shows the fuzzy rules for the decision of process priority and Fig. 7 represents the membership functions for it.

\section{Experimental Results}

For the experiments, we set three user groups of batch user group, interactive user group and real-time user group, each of which has 10 subjects. The experiments have been conducted by typing some given sentences using gedit, one of interactive processes, while watching movies using Mplayer, one of real-time processes. Each subject performed the experiments twice in the environments: Linux kernel 2.4.25 and the environment where the proposed method is applied. When the subjects typed the sentences, we ran 10 batch processes in background to overload the CPU.

For the user model, in the experiments, we designed three models for each class. We set the user's preference values, $\left(\omega_{\text {batch }}, \omega_{\text {inter }}, \omega_{r t}\right)=(0.7,0.15,0.15)$ for batch process user group, $(0.15,0.7,0.15)$ for interactive process user group and $(0.15,0.15$, 0.7 ) for real-time process user group.

While the subjects typing sentences, we saved the logs of each process in order to analyze the difference between normal Linux kernel and the proposed method, and after typing twice, the subjects evaluated the processes of their group about how good the proposed method was compared with the normal Linux kernel (1 5 points: 1 denotes bad while 5 denotes good); the batch user evaluated only the batch processes, the interactive user evaluated only the interactive process, gedit, and the real-time user evaluated only the real-time process, Mplayer.

Fig. 8 shows the result of user evaluation. The batch users gave 3.1 points in average which are lower than the other groups, and the interactive users gave 4.1 points which means they think the proposed method is better than normal method, and the real-time users gave 4.7 points which denotes that the proposed one is much better than normal one. The batch users gave low scores because the differences in batch processes are not able to confirm the performance intuitively by humans.

Fig. 9(a) shows the number of CPU occupations per unit time and Fig. 9(b) represents the CPU usage per unit time. Although the batch user group had less CPU occupation on batch processes than normal group (see Fig. 9(a)), it used 133.52 CPU

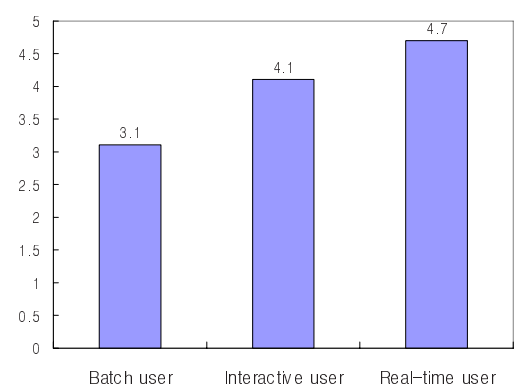

Fig. 8. The result of user evaluation 
time which was more than that of normal method which used 105.2 (see Fig. 9(b)). From this result, we can confirm that the proposed method has better performance on batch processes than normal method. However, the real-time user group used up 175.44 CPU time on batch processes which was more than batch user group. The realtime user group had much lower CPU occupation than the other user groups (see Fig. 9(a)). Therefore, there were lower overhead on saving logs that gave more CPU time to the running processes compared with the other groups.

Interactive user group has the most CPU time (0.32) on interactive processes compared with other groups and the second place of the CPU occupation (43.8), next to the real-time user group (47.97). Even though the interactive user group has more CPU time and occupations number than normal method, it does not explain sufficiently because interactive processes do not need much computational power and the CPU occupation depends on the speed of subject's typing. However, through user evaluation test (see Fig. 8), we can verify the proposed method of interactive user group is better on interactive processes.

In Fig. 9(a), the real-time user group has the highest CPU occupation on real-time processes (176.92). This denotes the real-time processes can get enough CPU time when it needs computation, so the number of broken images is less than that of normal method. The result of user evaluation also verifies the performance of realtime user group.

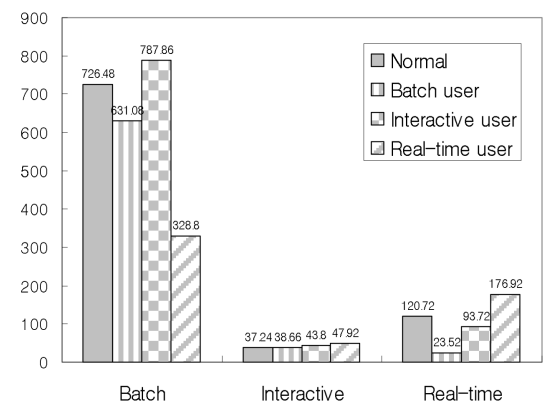

(a) No. of CPU occupations per unit time

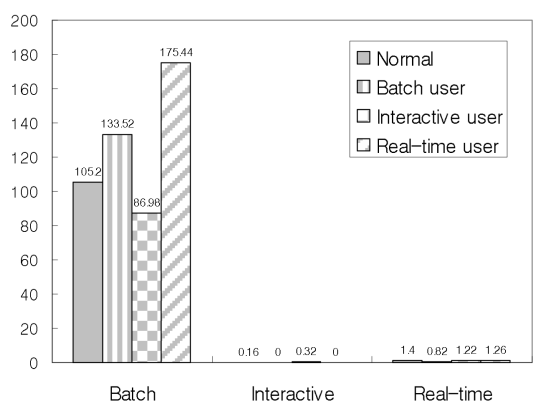

(b) CPU usage per unit time

Fig. 9. Experimental results

\section{Concluding Remarks}

In this paper, we have proposed a user adaptive process scheduling method using fuzzy inference. It classifies each process to batch process, interactive process and real-time process with extracted logs of processes, and it models the preferences of users. Finally it determines the nice value, the priority of the process, with the information of classifying processes and user preferences.

Compared with the conventional scheduling methods, the proposed method has three benefits. First, it can schedule processes according to the type of processes by classifying processes into three classes. Second, it provides adaptive scheduling method by modeling the user's preference. Third, it can provide different scheduling 
method to multi users without resetting operating systems. Although there is an overload to feedback when users log out, this is easier and simpler than compiling kernels to apply other scheduling methods. Moreover, the proposed method can support different scheduling methods to different users.

\section{Acknowledgments}

This research was supported by MIC, Korea under ITRC IITA-2006-(C1090-06030046).

\section{References}

1. Coulson, G., Blair, G.S., Grace, P.: On the performance of reflective systems software. In: Proc. Intl. Workshop on MP2004, Satellite workshop of the IEEE IPCCC2004 (2004)

2. Fassino, J.P., Stefani, J.B., Lawall, J., Muller, G.: THINK: A software framework for component-based operating system kernels. In: USENIX Annual Technical Conference (2002)

3. Bovet, D.P., Cesati, M.: Understanding the Linux Kernel. O’REILLY (2003)

4. Nieh, J., Vaill, C., Zhong, H.: Virtual-Time Round-Robin: An $\mathrm{O}(1)$ proportional share scheduler. In: USENIX Annual Technical Conference, pp. 245-260 (2001)

5. Stallings, W.: Operating Systems, Internals and Design Principles. Prentice Hall, Englewood Cliffs (2001)

6. Yavatkar, R., Lakshman, K.: A CPU scheduling algorithm for continuous media applications. In: Little, T.D.C., Gusella, R. (eds.) NOSSDAV 1995. LNCS, vol. 1018, pp. 210-213. Springer, Heidelberg (1995)

7. Nieh, J., Lam, M.S.: The design, implementation and evaluation of SMART: A scheduler for multimedia applications. In: Proc. of 16th ACM symposium on operating systems principles, pp. 184-197 (1997)

8. Bollella, G., Jeffay, K.: Support for real-time computing within general purpose operating systems. In: Proc. Of the Real-Time Technology and Applications Symposium, pp. 4-14 (1995)

9. Jones, M., Rosu, D., Rosu, M.: CPU reservations and time constraints: Efficient, predictable scheduling of independent activities. In: Proc. Of the 16th Symposium on Operating Systems Principles, pp. 198-211 (1997)

10. Duda, K., Cheriton, D.: Borrowed-virtual-time (BVT) scheduling: Supporting latencysensitive threads in a general-purpose scheduler. In: Proc. Of the 17th Symposium on Operating Systems Principles, pp. 261-276 (1999)

11. Evans, S., Clarke, K., Singleton, D., Smaalders, B.: Optimizing Unix resource scheduling for user interaction. USENIX Summer, pp. 205-218 (1993)

12. Setnes, M., Roubos, H.: GA-fuzzy modeling and classification: Complexity and performance. IEEE Trans. on Fuzzy Systems 8(5), 509-522 (2000)

13. Wu, H., Mendel, J.M.: Binary classification of ground vehicles based on the acoustic data using fuzzy logic rule-based classifiers. Technical Report 356, USC-SIPI (2002)

14. Roubos, J.A., Setnes, M., Abonyi, J.: Learning fuzzy classification rules from data. In: RASC conference (2000) 